\title{
REVIEW
}

\section{Mechanically induced osteogenic lineage commitment of stem cells}

\author{
Julia C Chen and Christopher R Jacobs ${ }^{*}$
}

\begin{abstract}
Bones adapt to accommodate the physical forces they experience through changes in architecture and mass. Stem cells differentiate into bone-forming osteoblasts, and mechanical stimulation is involved in this process. Various studies have applied controlled mechanical stimulation to stem cells and investigated the effects on osteogenic lineage commitment. These studies demonstrate that physical stimuli can induce osteogenic lineage commitment. Tension, fluid shear stress, substrate material properties, and cell shape are all factors that influence osteogenic differentiation. In particular, the level of tension is important. Also, rigid substrates with stiffness similar to collagenous bone induce osteogenic differentiation, while softer substrates induce other lineages. Finally, cells allowed to adhere over a larger area are able to differentiate towards the osteogenic lineage while cells adhering to a smaller area are restricted to the adipogenic lineage. Stem cells are able to sense their mechanical environments through various mechanosensors, including the cytoskeleton, focal adhesions, and primary cilia. The cytoskeleton provides a structural frame for the cell, and myosin interacts with actin to generate cytoskeletal tension, which is important for mechanically induced osteogenesis of stem cells. Adapter proteins link the cytoskeleton to integrins, which attach the cell to the substrate, forming a focal adhesion. A variety of signaling proteins are also associated with focal adhesions. Forces are transmitted to the substrate at these sites, and an intact focal adhesion is important for mechanically induced osteogenesis. The primary cilium is a single, immotile, antenna-like structure that extends from the cell into the extracellular space. It has emerged as an important signaling center, acting as a microdomain to facilitate biochemical signaling. Mechanotransduction is the process by which physical stimuli are converted into biochemical responses. When potential mechanosensors are disrupted, the activities of components of mechanotransduction pathways are also inhibited, preventing mechanically induced osteogenesis. Calcium, mitogen-activated protein kinase/extracellular signal-regulated kinase, Wnt, Yes-associated protein/transcriptional coactivator with PDZ-binding motif and RhoA/Rho kinase signaling are some of the mechanotransduction pathways proposed to be important. In this review, types of mechanical stimuli, mechanosensors, and key pathways involved in mechanically induced osteogenesis of stem cells are discussed.
\end{abstract}

\section{Introduction}

Bones are dynamic organs capable of adjusting their architecture and mass to withstand the physical forces they experience. Mechanotransduction, which is the process by which cells convert physical stimuli into biochemical responses, underlies this capability of bones. Mechanotransduction of stem cells has been studied through both inferential in vivo studies, and in vitro studies involving application of a controlled mechanical stimulation to cells.

\footnotetext{
* Correspondence: cri2111@columbia.edu

Department of Biomedical Engineering, Columbia University, New York, NY 10027, USA
}

There are many examples that demonstrate that bones adapt to their mechanical environment. One example of bones adapting to greater mechanical needs is that professional tennis players develop longer and denser bones in their dominant arms [1]. However, when mechanical demands are decreased, bone is lost, as seen during space travel, bed rest, or spinal cord injury. The adaptation response to mechanical load occurs not only in the mature skeleton, but begins very early during embryonic development [2-5]. The skeleton continues to adapt to its mechanical environment throughout life, although adaptation rates decrease with age [6].

The onset of involuntary muscle contractions in the embryo is correlated with the beginning of ossification, 
or creation of bone from rudiments. Mechanical stimulation is not required for initial bone formation, but formation is enhanced by the mechanical loads placed on bones by muscular contractions [2-5]. In early development, a majority of bone cells are directly derived from stem cells. Therefore, it is inferred that physical stimulation may promote osteogenic differentiation in vivo, and stem cells may be directly responsible for sensing and responding to physical stimulation.

In the adult, bone adaptation occurs throughout life. Bone apposition rates are elevated in response to increases in mechanical demands, and mechanical loading is thought to promote proliferation of stem cells, which then differentiate into bone-forming osteoblasts. Insights into the effect of physical stimulation on stem cells have also been obtained from surgical experiments. For example, osseointegration around an implant was enhanced by physical stimulation, suggesting that strain promotes osteogenic differentiation of stem cells [7]. Also, during distraction osteogenesis, where a fracture is created and bone segments are gradually moved apart, stem cells were observed to populate the zone between the bone segments. In addition, bone formation was associated with areas calculated to experience low to moderate tension $[8,9]$.

Due to the complicated mechanical environment and responses, however, it is difficult to determine the specific mechanisms by which stem cells may sense or respond to physical stimuli through in vivo studies alone. Therefore, studies applying controlled physical stimuli to cells in vitro are useful for uncovering potential molecular mechanisms for sensing mechanical stimulation and possible mechanotransduction pathways. Various studies have investigated the effect of stretch applied to stem cells either seeded within a matrix $[10,11]$, or on a flexible membrane [12-19]. Fluid flow as a means to apply shear stress to cells is also commonly used [20-25]. Bioreactors have also been used to produce a more complicated mechanical environment and to supply biochemical factors in a controlled manner over extended periods of time $[11,24,26]$.

Furthermore, mechanically induced bone formation occurs as a concerted response involving multiple cell types. The majority of cells in cortical bone are osteocytes, which are mature bone cells embedded within the calcified matrix. Other bone cells include osteoblasts, which are immature bone cells, bone lining cells, osteoclasts, and stem cells. Finally, endothelial and smooth muscle cells in the vasculature may also contribute to the response to loading [27]. Although most cells are able to sense their mechanical environment, osteocytes are thought to be the primary mechanosensing cell types in bone and are connected through processes extending through the lacuno-canalicular network. Through this network, osteocytes are capable of communicating to osteoblasts, bone lining cells, stem cells and osteoclasts through paracrine signaling to induce bone formation or resorption. However, recent in vitro studies have demonstrated that direct mechanical stimulation of stem cells also promotes osteogenic differentiation. While the focus of this review is restricted to stem cells, various recent reviews cover skeletal mechanobiology and the pathways involved [28], emphasizing the roles of osteocytes and cell-cell communication [27,29-31], osteoblasts [32] as well as bone lining cells [33].

In this review, mechanical signals, mechanosensors, and key pathways involved in mechanically induced osteogenic lineage commitment of stem cells are discussed.

\section{Physical stimuli}

Stem cells contributing to bone formation reside within the bone marrow and the periosteum, a thin fibrous membrane that surrounds the outer surface of all bones (Figure 1). Hydrostatic pressure and fluid flow-induced shear stress may be the dominant mechanical stimuli for mesenchymal stem cells (MSCs) residing within the bone marrow [34], whereas tension and compression are likely to be more important in the periosteum. During early development, cells within the rudiments also differentiate directly into bone cells. The effects of tension, compression, fluid shear stress, substrate material properties and cell shape on osteogenesis of stem cells are discussed here (Figure 2).

\section{Tension}

Tension has been observed to be an osteogenic stimulus for stem cells. In studies examining the effects of tension, stem cells are typically seeded on a flexible membrane or within a matrix to which strain is applied. However, differentiation is dependent on the manner in which strain is applied. Some factors that have been varied include magnitude, frequency, and application time. Results from separate studies are difficult to compare directly, but demonstrate

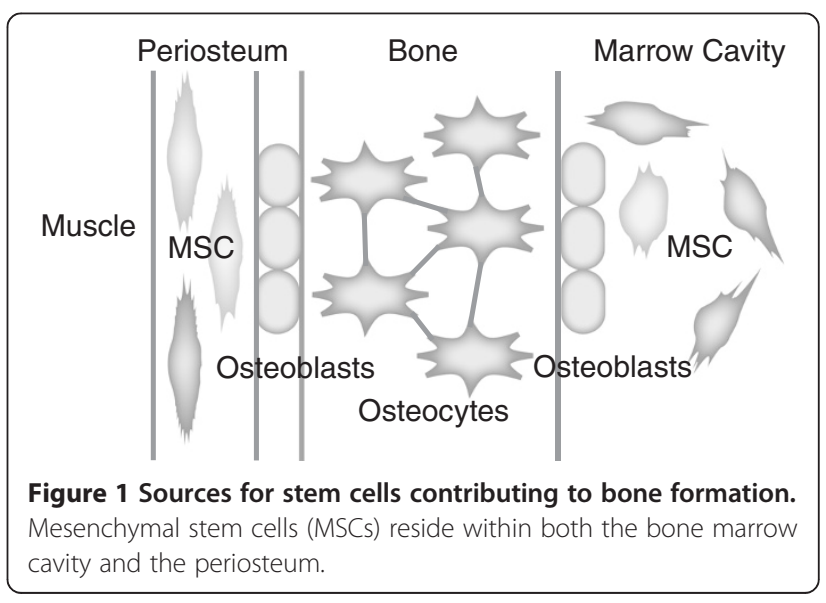




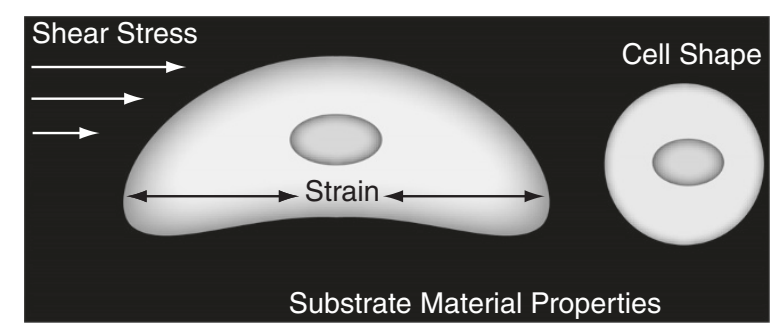

Figure 2 Cellular and pericellular mechanics. Strain, shear stress, substrate material properties and cell shape have been observed to affect osteogenesis of stem cells.

that tension can induce osteogenic differentiation, although the magnitude of tension applied varies between studies.

A study by Haasper and colleagues [12] applied cyclic stretch of 2 or $8 \%$ to human MSCs for 1 hour each day for 3 consecutive days. Modest increases in gene expression of Runx2, an osteogenic transcription factor, were observed and greater increases occurred with $8 \%$ tension compared to $2 \%$. Other groups have also confirmed that similar levels of tension induce osteogenic differentiation of MSCs. Application of tensile strains between 8 and 12\% resulted in reduced proliferation, as well as increased gene expression of bone markers, including Runx2 [13,35], alkaline phosphatase (ALP) [13,35], collagen type 1 (Col1) $[13,35]$, and bone morphogenetic protein (BMP)2 [10].

When mouse bone marrow stromal cells (ST2 cell line) were cultured in osteogenic media, however, proliferation increased when tension levels of 5 to $15 \%$ were applied [14]. In the same study, ALP activity and Runx2 gene expression also increased for strain levels $5 \%$ and below, but decreased with higher strains. In this case, higher strains were inhibitory for osteogenic differentiation. Similarly, other studies with both human and rat MSCs have also shown that low levels of tension promote osteogenic differentiation. In these studies, tensile strains from $0.2 \%$ to $5 \%$ were found to promote ALP activity [11,15-17], gene expression of osteogenic markers [11,15-19], and mineralized matrix deposition $[11,17,18]$. MSCs experiencing tension at these levels also exhibit reduced expression of adipogenic, chondrogenic, and neurogenic markers such as Col2, aggrecan, dystrophin related protein 2 , and peroxisome proliferator-activated receptor $\gamma$ [18]. However, the effect of low levels of tension on proliferation rates is unclear. In one study, proliferation rates were increased with application of $0.2 \%$ strain [15], while application of $2.5 \%$ strain resulted in decreased proliferation rates in another study [19]. Both studies used MSCs isolated from rat bone marrow.

\section{Compression}

Compression has been reported to result in both chondrogenic and osteogenic differentiation in human bone marrow-derived MSCs. Pelaez and colleagues [36] subjected MSCs seeded in a fibrin gel to dynamic mechanical compression with a maximum strain of $15 \%$ and observed increases in chondrogenic gene expression and deposition of sulfated glycosaminoglycans, indicating chondrogenic differentiation. Haudenschild and colleagues [37] also observed upregulation of genes associated with chondrogenesis in MSCs loaded with dynamic uniaxial unconfined compression. In the same study, tension was found to enhance expression of ossification-associated genes and inhibit chondrogenic gene expression. However, when Jagodzinski and colleagues [26] applied 10\% cyclic compression with continuous perfusion to MSCs, expression of Runx 2 and osteocalcin, a late stage bone marker, were increased, suggesting that the addition of perfusion to compression promoted osteogenic lineage commitment.

\section{Fluid shear stress}

Fluid flow is a commonly used method for applying fluid shear stress in vitro, producing a gradient of stresses along the cell body. The method of applying fluid shear stress can affect proliferation rates and differentiation. Directionality of fluid flow has been shown to be important, with cells experiencing unidirectional flow exhibiting different characteristics from cells experiencing oscillatory fluid flow. Other factors that have been examined include magnitude, frequency, and length of application.

Upon application of oscillatory fluid flow, MSCs exhibited immediate increases in intracellular calcium mobilization. Twenty-four hours after fluid flow, proliferation rates of human MSCs derived from bone marrow aspirate increased compared to static controls, and gene expression of osteopontin (OPN) and osteocalcin were also increased [20]. In another study, OPN gene expression also increased in mouse bone marrowderived MSCs exposed to oscillatory fluid flow for 3 hours at $1 \mathrm{~Hz}$, with a peak shear stress of $1.0 \mathrm{~Pa}$. Furthermore, DNA methylation of the OPN promoter also decreased, suggesting a change in epigenetic state. Changes in methylation are durable, and may affect chromatin architecture, which influences gene accessibility. This study demonstrated that mechanical stimulation in the form of fluid shear stress can induce epigenetic changes that promote osteogenic lineage commitment [21]. Mechanical stimulation in the form of mechanical strain combined with micropatterning to align cells can also induce epigenetic changes in human MSCs by affecting histone acetylation, which is important for chromatin architecture [22].

In a longer term study, human bone marrow-derived MSCs were cultured on either glass or calcium phosphatecoated glass and exposed to uniform fluid shear stress of $0.0012 \mathrm{~Pa}$ for 10 days. Exposure to fluid flow resulted in reduced proliferation, promoted Col1 and mineralized 
matrix deposition, and increased mRNA levels of the osterix bone transcription factor. OPN and bone sialoprotein mRNA levels were dependent on the type of substrate that cells were seeded on [23].

The effect of fluid flow in a three-dimensional environment has also been tested using perfusion bioreactors. Rat bone marrow-derived MSCs were seeded into porous scaffolds and cultured for 15 days under pulsatile fluid flow at various frequencies. ALP activity, mRNA levels of OPN, and accumulation of OPN and prostaglandin $E_{2}$ were all enhanced with perfusion relative to static culture conditions [24].

Hydrostatic pressure can also encourage osteogenic differentiation. Both static $(23 \mathrm{kPa})$ or dynamic hydrostatic pressures (10 to $36 \mathrm{kPa}, 0.25 \mathrm{~Hz}$ ) were capable of inducing osteogenesis in rat bone marrow-derived MSCs [25].

\section{Substrate material properties}

The material properties of the substrate that cells are seeded upon may also affect osteogenic differentiation. More rigid surfaces were found to induce osteogenic lineage commitment for TG2 $\alpha$ E14 mouse embryonic stem cells [38]. Human MSCs seeded on substrates with elasticity similar to collagenous bone (25 to $40 \mathrm{kPa})$ developed an osteoblast-like morphology and upregulated Run $x 2$ gene expression, whereas cells seeded on softer substrates developed other morphologies and upregulated transcription factors important for other lineages. Elasticitydirected lineage specification was found to be dependent on nonmuscle myosin II, which may exert force through focal adhesions [39]. In studies with rat kidney epithelial cells and mouse fibroblasts [40] or with rat aorta-derived smooth muscle cells [41], the cytoskeleton and focal adhesions were found to be affected by the underlying substrate, thereby altering cell shape and the internal tension generated by the cell. MSCs may also use a similar mechanism, as the application of blebbistatin to block nonmuscle myosin II, which is thought to exert force through focal adhesions, prevents differentiation [39].

\section{Cell shape}

The shape of a cell can also affect its differentiation potential. McBeath and colleagues [42] used micropatterning techniques to create islands of varying sizes to restrict the area over which human MSCs adhere. Cells seeded on larger islands were observed to differentiate towards the osteogenic lineage, whereas those on small islands were restricted to the adipogenic lineage. High levels of RhoA, a GTPase that regulates contractility, and its effector Rho kinase (ROCK) were correlated with osteogenic conditions. Constitutively active RhoA also caused osteogenesis and required actin-myosin-generated tension.

Gradients of mechanical forces can also drive differentiation. Micropatterning techniques were used to confine monolayers of human MSCs within various shapes with convex and concave edges [43]. Cells seeded at convex edges exhibited greater positive staining for ALP than those at concave edges, which exhibited increased staining for lipid droplets. Cell traction forces at the convex edges were also greater than those at the concave edges. Therefore, higher traction forces were correlated with osteogenesis. Furthermore, osteogenesis also required myosin-generated tension while adipogenesis did not.

\section{Disuse}

Finally, the absence of mechanical stimulation also affects stem cell differentiation. When sciatic neurectomies were performed on rats, producing immobilization and effectively reducing mechanical loading on bones, the number of adherent cells obtained from bone marrow isolation was reduced $50 \%$. This suggests that a decrease in mechanical loading is associated with decreased numbers of MSCs, the primary cell type in the adherent population. Furthermore, the osteogenic potential of MSCs from unloaded bones is diminished compared with loaded bones, as indicated by decreased ALP activity and reduced nodule formation [44]. Similar results were seen in another study utilizing tail suspension in rats to reduce loading in the hindlimbs. Again, the total adherent marrow stromal cell population was decreased, and only half the normal number of colonies was formed. Proliferation of ALP-positive cells was also inhibited, and OPN gene expression was reduced [45]. Disuse due to skeletal unloading by hindlimb suspension in rats has been demonstrated to bias commitment towards the adipogenic lineage [46]. However, mice treated with low-magnitude whole body vibrations during hindlimb suspension had a greater population of osteogenic marrow stromal cells, suggesting that osteogenic potential was retained [47].

The mechanical environment of stem cells in vivo is complicated. However, in vitro studies described above applying tension, compression, and fluid shear stress, or controlling substrate material properties and cell shape demonstrate that stem cells are responsive to these mechanical stimuli.

\section{Mechanosensors}

Various mechanosensors have been proposed through which stem cells may sense the mechanical environment. These include the cytoskeleton, focal adhesions, primary cilia, membrane channels, gap junctions and mechanosomes $[28,48,49]$. A complicated picture is emerging, including involvement of multiple mechanosensors and the biochemical pathways activated by each during osteogenesis. The cytoskeleton, focal adhesions, and primary cilia are described in more detail below (Figure 3). 


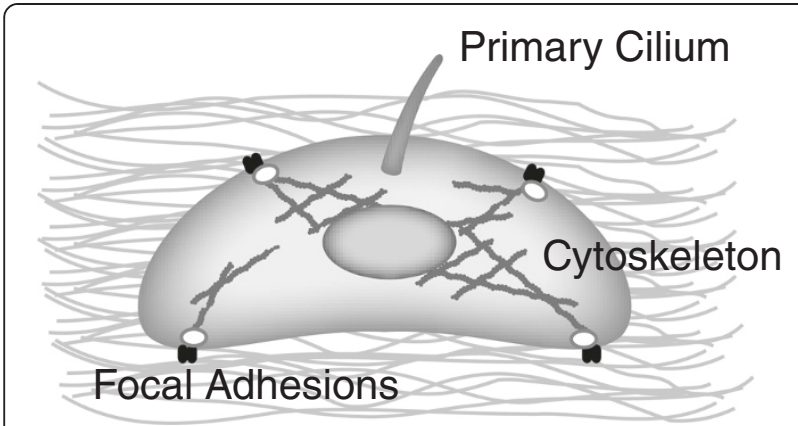

Figure 3 Mechanosensors. The cytoskeleton, focal adhesions and primary cilium are potentially important in stem cells for detecting and responding to physical stimuli.

\section{Cytoskeleton}

The cytoskeleton provides a structural frame for the cell and is composed primarily of actin, intermediate filaments, and microtubules. Myosin interacts with actin to generate cytoskeletal tension, which is important for mechanically induced osteogenesis of human MSCs [42]. In addition to an intact cytoskeleton, RhoA, a GTPase, and its effector, ROCK, regulate cytoskeletal dynamics and were required for fluid flow-induced osteogenic differentiation of mouse MSCs (C3H10T1/2 line). Disruption of the cytoskeleton through inhibition of nonmuscle myosin II, actin polymerization, and actin depolymerization was observed to prevent fluid flow-induced osteogenic differentiation. Furthermore, disruption of the cytoskeleton also resulted in differentiation towards the adipogenic and chon-drogenic lineages to a greater extent [50]. The total traction force per cell has also been correlated with cell spread area in human embryonic stem cells. Cytoskeletal contractility, expression of E-cadherin and distribution of focal adhesions may together influence mechanically induced differentiation of stem cells [51].

However, the role of the cytoskeleton in mechanosensing may be different in three-dimensional conditions. Although osteogenesis was enhanced in human bone marrow stromal cells cultured in gels with increasing matrix stiffness, cells lacked well-defined actin filaments. Disruption of cytoskeletal structure using small molecule inhibitors also did not affect differentiation, although integrinextracellular matrix interactions were required [52].

\section{Focal adhesions}

Adapter proteins such as talin and vinculin link the cytoskeleton to integrins, which attach the cell to the substrate, forming a focal adhesion. Integrins are composed of two subunits, alpha and beta, with various isotypes of each. A variety of signaling proteins are also associated with focal adhesions, including focal adhesion kinase (FAK), an important mediator of signaling at these centers. Forces are also transmitted to the substrate at these sites. In fibroblasts, local forces correlate with the area of focal adhesions, and the blocking of actomyosin contractility using butanedione monoxime resulted in rapid disruption of focal adhesions [53].

The $\beta 1$ integrin has been shown to be important for mediating the response of human bone marrow-derived MSCs to mechanical stimulation [54]. Upon application of fluid shear stress, an increase in ALP activity and expression of osteogenic markers were observed, along with activation of FAK and extracellular signal-regulated kinase 1/2 (ERK1/2). However, when $\beta 1$ integrins were blocked through RGDS peptides or antibodies, FAK and ERK1/2 activation were inhibited [54]. Phosphorylation of FAK has also been demonstrated to be important for osteogenic differentiation of human MSCs in response to tension [55].

However, the roles of FAK and integrins in vivo may differ from their roles in vitro. Fibroblasts (NIH-3 T3 mouse) cultured in three dimensions displayed different integrin content, and decreased phosphorylation of FAK compared to cells cultured in two dimensions [56]. Despite these reported differences, FAK has been demonstrated to be important for mechanically induced osteogenesis in vivo. Physical stimulation of a device implanted into bone resulted in Runx2 expression in progenitor cells located at a greater distance from the device than when the device remained stationary. However, conditional inactivation of FAK in cells expressing the osteoblast-specific Colla1 promoter abolished this mechanically induced osteogenic response [57].

\section{Primary cilia}

The primary cilium is a single, immotile, antenna-like structure that extends from the cell into the extracellular space [58]. Once thought to be a vestigial structure, the primary cilium has emerged as an important signaling center and has been demonstrated to be important for mechanosensation in multiple cell types. The primary cilium acts as a microdomain, facilitating biochemical signaling as a result of localization and concentration of various proteins, including ion channels [59] and enzymes [60]. Primary cilia are important for normal bone development [61], and for sensing a variety of extracellular biochemical [62] and biophysical signals, including fluid shear stress in MC3T3-E1 osteoblast- and MLO-Y4 osteocytelike cells [63]. Fluid flow-induced paracrine signaling in bone may also be primary cilia dependent. In response to fluid flow, MLO-Y4 osteocyte-like cells released factors that upregulated OPN gene expression in C3H10T1/2 cells, a mouse MSC line. However, when primary cilia formation was inhibited in the osteocyte-like cells, the flowinduced changes in MSC osteogenic gene expression did not occur [64]. 
Recently, primary cilia have also been demonstrated to have a direct role in mechanically activated signaling in human MSCs. Exposure to fluid flow resulted in an increase in cyclooxygenase 2 and BMP2 gene expression, indicating an early osteogenic response to mechanical stimulation. A significant increase in proliferation rate was also observed. However, human MSCs treated with small interfering RNA to inhibit intraflagellar transport 88, an intraflagellar transport protein important for primary cilia formation, did not respond with the flowinduced increases in gene expression. Interestingly, cells with impeded primary cilia formation also responded to flow with an increase in proliferation rate [65]. These results suggest primary cilia are important for mechanically induced osteogenic differentiation.

\section{Mechanotransduction pathways}

Multiple pathways mediate osteogenic lineage commitment. The role of calcium signaling, and the mitogenactivated protein kinase (MAPK)/ERK, Wnt, Hippo, and RhoA/ROCK pathways are discussed in more detail below.

Calcium is a major second messenger that affects many pathways. Human MSCs upregulate and downregulate the frequency of calcium transients upon application of a $20 \%$ static uniaxial compressive strain for 20 minutes [66]. Exposure of human bone marrow-derived MSCs to oscillatory fluid flow also resulted in dramatic transient increases in intracellular calcium levels, with the first responses occurring approximately 10 seconds after the start of flow [20]. Changes in intracellular calcium levels are an immediate response to mechanical stimulation, and can activate downstream signaling pathways.

Mechanically induced increases in osteogenic gene expression can involve various components of the MAPK/ ERK pathway. When strain was applied to MSCs, phosphorylation levels of ERK1/2 were elevated, and osteogenic differentiation was dependent on ERK1/2 activity [35]. Tension-mediated increases in mineralization also required activation of ERK1/2, with inhibition of MEK (mitogen-activated protein kinase kinase) blocking these increases in human MSCs [18]. Inhibition of ERK, p38, and pI3 kinases also reduced BMP2 expression in rat MSCs isolated from bone marrow [19]. Furthermore, the inhibition of stretch-activated cation channels with gadolinium chloride also reduced Col1 expression, suggesting that calcium activity and MAPK/ERK signaling together were required for an osteogenic response in this case [19].

Wnt signaling plays an important role in bone development, but its role in MSC lineage commitment is unclear. The canonical pathway involves the translocation of $\beta$-catenin to the nucleus, and $\beta$-catenin has been shown to promote osteogenic differentiation in early osteoblast progenitors in vivo [67]. In contrast, other studies have suggested that canonical Wnt signaling may actually promote stem cell renewal and inhibit osteogenic differentiation of osteoprogenitor cells in vivo [68], as well as promote stem cell renewal in human MSCs derived from bone marrow [69]. Arnsdorf and colleagues [70] investigated the role of non-canonical Wnt signaling in mechanically induced osteogenic differentiation of C3H10T1/2 mouse MSCs. Exposure of MSCs to oscillatory fluid flow resulted in translocation of $\beta$-catenin and upregulation of Wnt5a, which is capable of inducing both canonical and non-canonical pathways. Wnt5a is also necessary for the flow-induced activation of RhoA and increase in Runx2 gene expression. However, inhibition of Wnt5a did not affect $\beta$-catenin translocation, which may be regulated instead by cadherin-catenin signaling. This study demonstrates that non-canonical Wnt signaling is important for mechanically induced differentiation in vitro [70].

Yes-associated protein (YAP) and transcriptional coactivator with PDZ-binding motif (TAZ) are effectors of the Hippo pathway and, like $\beta$-catenin, also translocate to the nucleus and regulate gene expression [71]. The YAP/TAZ pathway is important for sensing substrate stiffness and cell shape. This pathway was also shown to be necessary for osteogenic differentiation of human bone marrow-derived MSCs, with depletion of YAP and TAZ inhibiting osteogenic differentiation of MSCs seeded on stiff extracellular matrix and large islands, which would normally promote osteogenic differentiation [72].

RhoA, a small GTPase, and its effector protein, ROCK, affect myosin-generated cytoskeletal tension and are important in human MSC lineage commitment along the adipogenic, chondrogenic and osteogenic pathways [42]. When C3H10T1/2 mouse MSCs were exposed to oscillatory fluid flow, RhoA and ROCK were activated. Application of lysophosphatidic acid sodium salt to increase activation of RhoA increased cytoskeletal tension, and further enhanced the flow-induced upregulation of Runx2, suggesting that RhoA and flow act synergistically. However, inhibition of ROCK, and disruption of cytoskeletal tension prevented the flow-induced upregulation of Runx2 [50].

\section{Perspectives on current understanding}

While many studies have found that MSCs are responsive to mechanical stimulation, there is not a consensus on how these mechanical factors should be applied to promote osteogenic differentiation. Tension and fluid shear stress are commonly used, but the resulting effects are dependent on the manner in which they are applied. For example, while some studies have found high levels 
of tension to be osteogenic $[10,12,13,35]$, others have found these levels to be inhibitory with low tension being osteogenic instead [11,14-19]. The profile of fluid flow has also been varied between studies, although steady [23], pulsatile [24], or oscillatory [20,21] fluid shear stress have all been shown to promote osteogenic differentiation of MSCs. However, oscillating flow is much less potent (fraction of cells responding, and amplitude of response) at stimulating bone cells than steady or pulsatile flow [73], and stress fibers form earlier with steady fluid flow [74].

Potential mechanosensors have been described above and are involved in sensing different types of physical stimuli, and do not necessarily act independently. For example, cytoskeletal tension is required for mechanotransduction with fluid flow in osteoblasts [63]. A fully functional cytoskeleton is also required for hypertrophy of the periosteum under three-point bending, as inhibition of ROCK, which is important for actin remodeling, reduces loading-induced hypertrophy [75]. Focal adhesions also act in conjunction with the cytoskeleton. Focal adhesions are connected to the cytoskeleton through adapter proteins such as talin and vinculin, which link integrins to the cytoskeleton. Tenogenic gene expression due to stretch was observed to be attenuated in human MSCs when ROCK, actin polymerization, and FAK were inhibited [76]. While not specific to osteogenic gene expression, this indicates that these pathways can interact. The primary cilium also influences the structure of the local cytoskeleton. Upon mechanical stimulation of MSCs with oscillatory fluid flow, the number of microtubules at the base of primary cilia increased [77]. In renal epithelial cells, fluid shear stress induces a primary cilia-mediated increase in intracellular calcium. However, inhibiting microtubule formation, actomyosin tension generation, and integrin attachment to the extracellular matrix inhibited the response to calcium. This indicates that mechanotransduction through primary cilia are not independent of the cytoskeleton and focal adhesions in these cells [78].

The mechanotransduction pathways described here are also shared among different forms of mechanical stimulation. Calcium, MAPK/ERK, Wnt and RhoA/ROCK pathways are involved in mechanotransduction under both strain and oscillatory fluid flow, and these pathways can also interact. For example, when uniaxial tension was applied to rat tendon-derived stem cells, Wnt5a and RhoA were both shown to influence osteogenic marker expression [79]. YAP and TAZ also interact with $\beta$-catenin in the Wnt pathway and components of the transforming growth factor- $\beta$ signaling pathway [71].

While mechanotransduction of more mature cell types has been extensively studied, the mechanobiology of stem cells remains understudied. As a consequence of the relatively few studies published, it is currently difficult to directly compare between studies using different cell types or types of mechanical stimulation. Certainly, it is unclear what the optimal method of mechanical stimulation is for osteogenic differentiation. Furthermore, the number of mechanotransduction pathways investigated in osteogenic differentiation of stem cells is limited. There are many potential future directions in research, and continued effort in this area could lead to important advancements in the treatment of bone-related diseases.

The in vivo mechanical microenvironments that stem cells exist within are complicated. In vitro studies utilize a simplified system to test the effects of various mechanical factors, and many insights have been gained through these experiments. However, the mechanical environment and the ability of cells to sense mechanical cues within the body may be very different from the in vitro situation. As mentioned above, fibroblasts display matrix adhesions in three dimensions that differ in structure, localization and function compared to the focal and fibrillar adhesions formed when cultured in two dimensions [56]. Future in vivo studies with stem cell-specific inhibitions or activations of mechanosensor components or mechanotransduction pathways could be very important for the development of novel therapeutics.

Another area in need of future investigations is the role of stem cell niches. The niche, which is the microenvironment of the cell and includes biochemical factors, is also important in directing stem cell differentiation. Interestingly, similar forms of mechanical stimulation can promote cell fate specification along different lineages. For example, 1 Pa fluid shear stress can induce cardiomyogenic [80], endothelial [81], or osteogenic differentiation [21]. Biophysical stimulation certainly plays a role in enhancing or inhibiting differentiation. In order for biophysical stimulation to promote lineage-specific differentiation, however, additional signals derived from the stem cell niche are necessary. Determining the roles of these signals could also lead to optimal differentiation of stem cells.

Finally, epigenetic changes that are induced by mechanical signals should be considered. Epigenetic changes refer to alterations of DNA that can activate or silence genes without changing DNA sequence. Methylation of cytosines in a CpG dinucleotide and histone modifications are commonly studied, and both of these change the accessibility of DNA for gene expression. Epigenetic state is durable and heritable, and is important for lineage commitment. Active research in epigenetics in recent years has resulted in many advances. Although very few studies have investigated epigenetic changes in mechanically induced osteogenic differentiation of stem cells, changes in DNA methylation have been detected [21]. This indicates that epigenetic changes do occur in this context, and other types of epigenetic changes could also be investigated. 


\section{Conclusion}

Many studies have been described in this review that demonstrate that physical stimuli can induce osteogenic lineage commitment in stem cells. In particular, tension, fluid shear stress, substrate material properties and cell shape are discussed here. The mechanism by which cells sense their mechanical environment is unclear, but various potential mechanosensors have been implicated. The cytoskeleton, focal adhesions and primary cilia have all been shown to be important for mechanically induced osteogenesis as discussed above. Physical stimuli are then transduced into biochemical responses through mechanotransduction pathways. Important components and pathways for mechanically induced osteogenic differentiation include calcium signaling, and the MAPK/ERK, Wnt, YAP/TAZ and RhoA/ROCK pathways.

Regenerative medicine holds the promise of replacing tissues or organs that have been lost or damaged. Many approaches involve the use of stem cells, as they are capable of proliferating, providing a large pool of cells to differentiate into the mature cell types required for the replacing tissue. In addition, induced pluripotent stem cells are increasingly studied and are a potentially accessible source of cells. The outlook on regenerative medicine in treating various bone-related diseases is very promising. It is clear from the studies described here that mechanical stimulation is an important factor for the osteogenic differentiation of stem cells. However, much still remains unclear about the nature of mechanical stimulation experienced by stem cells, the roles of mechanosensors, and biochemical pathways activated. Future research could also include more in vivo studies, and investigate the role of the stem cell niche and epigenetics. Studies in these exciting areas could yield valuable insights for the treatment of bone diseases.

\section{Note: This article is part of a thematic series on Physical influences on stem cells edited by Gordana Vunjak-Novakovic. Other articles in the series can be found online at http://stemcellres.com/series/ physical}

\begin{abstract}
Abbreviations
ALP: Alkaline phosphatase; BMP: Bone morphogenetic protein; Col: Collagen; ERK1/2: Extracellular signal-regulated kinase 1/2; FAK: Focal adhesion kinase; MAPK: Mitogen-activated protein kinase; MSC: Mesenchymal stem cell; OPN: Osteopontin; ROCK: Rho kinase; TAZ: Transcriptional coactivator with PDZ-binding motif; YAP: Yes-associated protein
\end{abstract}

\section{Competing interests}

Both authors declare that they have no competing interests.

\section{Authors' contributions}

JCC and CRJ drafted the manuscript. Both authors read and approved the final manuscript.

\section{Acknowledgements}

This work was supported by a New York State Stem Cell Grant (N089-210) and $\mathrm{NIH}$ grants (AR45989, AR54156).

Published: 4 September 2013

\section{References}

1. Krahl H, Michaelis U, Pieper HG, Quack G, Montag M: Stimulation of bone growth through sports. A radiologic investigation of the upper extremities in professional tennis players. Am J Sports Med 1994, 22:751-757.

2. Hall BK, Herring SW: Paralysis and growth of the musculoskeletal system in the embryonic chick. J Morphol 1990, 206:45-56.

3. Dietz FR: Effect of denervation on limb growth. J Orthop Res 1989, 7:292-303.

4. Rodriguez Jl, Garcia-Alix A, Palacios J, Paniagua R: Changes in the long bones due to fetal immobility caused by neuromuscular disease. A radiographic and histological study. J Bone Joint Surg Am 1988, 70:1052-1060.

5. Rodriguez JI, Palacios J, Garcia-Alix A, Pastor I, Paniagua R: Effects of immobilization on fetal bone development. A morphometric study in newborns with congenital neuromuscular diseases with intrauterine onset. Calcif Tissue Int 1988, 43:335-339.

6. van der Meulen MC, Beaupre GS, Carter DR: Mechanobiologic influences in long bone cross-sectional growth. Bone 1993, 14:635-642.

7. Leucht P, Kim JB, Wazen R, Currey JA, Nanci A, Brunski JB, Helms JA: Effect of mechanical stimuli on skeletal regeneration around implants. Bone 2007, 40:919-930

8. Carter DR, Beaupre GS, Giori NJ, Helms JA: Mechanobiology of skeletal regeneration. Clin Orthop Relat Res 1998:S41-S55.

9. Tay BK, Le AX, Gould SE, Helms JA: Histochemical and molecular analyses of distraction osteogenesis in a mouse model. J Orthop Res 1998, 16:636-642.

10. Sumanasinghe RD, Bernacki SH, Loboa EG: Osteogenic differentiation of human mesenchymal stem cells in collagen matrices: effect of uniaxial cyclic tensile strain on bone morphogenetic protein (BMP-2) mRNA expression. Tissue Eng 2006, 12:3459-3465.

11. Mauney JR, Sjostorm S, Blumberg J, Horan R, O'Leary JP, Vunjak-Novakovic G, Volloch V, Kaplan DL: Mechanical stimulation promotes osteogenic differentiation of human bone marrow stromal cells on 3-D partially demineralized bone scaffolds in vitro. Calcif Tissue Int 2004, 74:458-468.

12. Haasper $\mathrm{C}$, Jagodzinski M, Drescher M, Meller R, Wehmeier M, Krettek C, Hesse E: Cyclic strain induces FosB and initiates osteogenic differentiation of mesenchymal cells. Exp Toxicol Pathol 2008, 59:355-363.

13. Jagodzinski M, Drescher M, Zeichen J, Hankemeier S, Krettek C, Bosch U, van Griensven M: Effects of cyclic longitudinal mechanical strain and dexamethasone on osteogenic differentiation of human bone marrow stromal cells. Eur Cells Mat 2004, 7:35-41. discussion 41.

14. Koike M, Shimokawa H, Kanno Z, Ohya K, Soma K: Effects of mechanical strain on proliferation and differentiation of bone marrow stromal cell line ST2. J Bone Miner Metab 2005, 23:219-225.

15. Qi MC, Hu J, Zou SJ, Chen HQ, Zhou HX, Han LC: Mechanical strain induces osteogenic differentiation: Cbfa1 and Ets-1 expression in stretched rat mesenchymal stem cells. Int J Oral Maxillofac Surg 2008, 37:453-458

16. Friedl G, Schmidt H, Rehak I, Kostner G, Schauenstein K, Windhager R Undifferentiated human mesenchymal stem cells (hMSCs) are highly sensitive to mechanical strain: transcriptionally controlled early osteochondrogenic response in vitro. Osteoarthritis Cartilage 2007, 15:1293-1300.

17. Huang $\mathrm{CH}$, Chen $\mathrm{MH}$, Young $\mathrm{TH}$, Jeng JH, Chen YJ: Interactive effects of mechanical stretching and extracellular matrix proteins on initiating osteogenic differentiation of human mesenchymal stem cells. J Cell Biochem 2009, 108:1263-1273.

18. Ward DF Jr, Salasznyk RM, Klees RF, Backiel J, Agius P, Bennett K, Boskey A, Plopper GE: Mechanical strain enhances extracellular matrix-induced gene focusing and promotes osteogenic differentiation of human mesenchymal stem cells through an extracellular-related kinasedependent pathway. Stem Cells Dev 2007, 16:467-480.

19. Kearney EM, Farrell E, Prendergast PJ, Campbell VA: Tensile strain as a regulator of mesenchymal stem cell osteogenesis. Ann Biomed Eng 2010, 38:1767-1779

20. Li YJ, Batra NN, You L, Meier SC, Coe IA, Yellowley CE, Jacobs CR: Oscillatory fluid flow affects human marrow stromal cell proliferation and differentiation. J Orthop Res 2004, 22:1283-1289. 
21. Arnsdorf EJ, Tummala P, Castillo AB, Zhang F, Jacobs CR: The epigenetic mechanism of mechanically induced osteogenic differentiation. J Biomech 2010, 43:2881-2886.

22. Li Y, Chu JS, Kurpinski K, Li X, Bautista DM, Yang L, Sung KL, Li S: Biophysical regulation of histone acetylation in mesenchymal stem cells. Biophys $J$ 2011, 100:1902-1909.

23. Scaglione S, Wendt D, Miggino S, Papadimitropoulos A, Fato M, Quarto R, Martin I: Effects of fluid flow and calcium phosphate coating on human bone marrow stromal cells cultured in a defined 2D model system. J Biomed Mater Res A 2008, 86:411-419.

24. Kavlock KD, Goldstein AS: Effect of pulse frequency on the osteogenic differentiation of mesenchymal stem cells in a pulsatile perfusion bioreactor. J Biomech Eng 2011, 133:091005.

25. Liu J, Zhao Z, Li J, Zou L, Shuler C, Zou Y, Huang X, Li M, Wang J: Hydrostatic pressures promote initial osteodifferentiation with ERK1/2 not p38 MAPK signaling involved. J Cell Biochem 2009, 107:224-232.

26. Jagodzinski M, Breitbart A, Wehmeier M, Hesse E, Haasper C, Krettek C, Zeichen J, Hankemeier S: Influence of perfusion and cyclic compression on proliferation and differentiation of bone marrow stromal cells in 3dimensional culture. J Biomech 2008, 41:1885-1891.

27. Rubin J, Rubin C, Jacobs CR: Molecular pathways mediating mechanical signaling in bone. Gene 2006, 367:1-16.

28. Liedert A, Claes L, lgnatius A: Signal transduction pathways involved in mechanotransduction in osteoblastic and mesenchymal stem cells. In Mechanosensitive Ion Channels. Edited by Kamkin A, Kiseleva I. Springer; 2008:253-265.

29. Castillo $A B$, Jacobs CR: Skeletal mechanobiology. In Mechanobiology Handbook. Edited by Nagatomi J. Boca Raton, FL: CRC Press; 2010:179-206.

30. Klein-Nulend J, Bacabac RG, Bakker AD: Mechanical loading and how it affects bone cells: the role of the osteocyte cytoskeleton in maintaining our skeleton. Eur Cells Mater 2012, 24:278-291.

31. Robling AG: The interaction of biological factors with mechanical signals in bone adaptation: recent developments. Curr Osteoporos Rep 2012, 10:126-131.

32. Papachroni KK, Karatzas DN, Papavassiliou KA, Basdra EK, Papavassiliou AG: Mechanotransduction in osteoblast regulation and bone disease. Trends Mol Med 2009, 15:208-216.

33. Robling $A G$, Turner $\mathrm{CH}$ : Mechanical signaling for bone modeling and remodeling. Crit Rev Eukaryot Gene Expr 2009, 19:319-338.

34. Gurkan UA, Akkus O: The mechanical environment of bone marrow: a review. Ann Biomed Eng 2008, 36:1978-1991.

35. Zhang $P$, Wu $Y$, Jiang $Z$, Jiang $L$, Fang B: Osteogenic response of mesenchymal stem cells to continuous mechanical strain is dependent on ERK1/2-Runx2 signaling. Int J Mol Med 2012, 29:1083-1089.

36. Pelaez D, Arita N, Cheung HS: Extracellular signal-regulated kinase (ERK) dictates osteogenic and/or chondrogenic lineage commitment of mesenchymal stem cells under dynamic compression. Biochem Biophys Res Commun 2012, 417:1286-1291.

37. Haudenschild AK, Hsieh AH, Kapila S, Lotz JC: Pressure and distortion regulate human mesenchymal stem cell gene expression. Ann Biomed Eng 2009, 37:492-502.

38. Evans ND, Minelli C, Gentleman E, LaPointe V, Patankar SN, Kallivretaki M, Chen X, Roberts CJ, Stevens MM: Substrate stiffness affects early differentiation events in embryonic stem cells. Eur Cells Mater 2009, 18:1-13. discussion 13-14.

39. Engler AJ, Sen S, Sweeney HL, Discher DE: Matrix elasticity directs stem cell lineage specification. Cell 2006, 126:677-689.

40. Pelham RJ Jr, Wang Y: Cell locomotion and focal adhesions are regulated by substrate flexibility. Proc Natl Acad Sci U S A 1997, 94:13661-13665.

41. Engler A, Bacakova L, Newman C, Hategan A, Griffin M, Discher D: Substrate compliance versus ligand density in cell on gel responses. Biophys J 2004, 86:617-628

42. McBeath R, Pirone DM, Nelson CM, Bhadriraju K, Chen CS: Cell shape, cytoskeletal tension, and RhoA regulate stem cell lineage commitment. Dev Cell 2004, 6:483-495.

43. Ruiz SA, Chen CS: Emergence of patterned stem cell differentiation within multicellular structures. Stem Cells 2008, 26:2921-2927.

44. Keila S, Pitaru S, Grosskopf A, Weinreb M: Bone marrow from mechanically unloaded rat bones expresses reduced osteogenic capacity in vitro. J Bone Miner Res 1994, 9:321-327.

45. Zhang R, Supowit SC, Klein GL, Lu Z, Christensen MD, Lozano R, Simmons $D J$ : Rat tail suspension reduces messenger RNA level for growth factors and osteopontin and decreases the osteoblastic differentiation of bone marrow stromal cells. J Bone Miner Res 1995, 10:415-423.
46. Ahdjoudj S, Lasmoles F, Holy X, Zerath E, Marie PJ: Transforming growth factor beta2 inhibits adipocyte differentiation induced by skeletal unloading in rat bone marrow stroma. J Bone Miner Res 2002, 17:668-677.

47. Ozcivici E, Luu YK, Rubin CT, Judex S: Low-level vibrations retain bone marrow's osteogenic potential and augment recovery of trabecular bone during reambulation. PLOS One 2010, 5:e11178.

48. Delaine-Smith RM, Reilly GC: The effects of mechanical loading on mesenchymal stem cell differentiation and matrix production. Vitam Horm 2011, 87:417-480.

49. Pavalko FM, Norvell SM, Burr DB, Turner CH, Duncan RL, Bidwell JP: A model for mechanotransduction in bone cells: the load-bearing mechanosomes. J Cell Biochem 2003, 88:104-112.

50. Arnsdorf EJ, Tummala P, Kwon RY, Jacobs CR: Mechanically induced osteogenic differentiation - the role of RhoA, ROCKII and cytoskeletal dynamics. J Cell Sci 2009, 122:546-553.

51. Sun Y, Villa-Diaz LG, Lam RH, Chen W, Krebsbach PH, Fu J: Mechanics regulates fate decisions of human embryonic stem cells. PLoS One 2012, 7:e37178.

52. Parekh SH, Chatterjee K, Lin-Gibson S, Moore NM, Cicerone MT, Young MF, Simon CG Jr: Modulus-driven differentiation of marrow stromal cells in 3D scaffolds that is independent of myosin-based cytoskeletal tension. Biomaterials 2011, 32:2256-2264.

53. Balaban NQ, Schwarz US, Riveline D, Goichberg P, Tzur G, Sabanay I, Mahalu D, Safran S, Bershadsky A, Addadi L, Geiger B: Force and focal adhesion assembly: a close relationship studied using elastic micropatterned substrates. Nat Cell Biol 2001, 3:466-472.

54. Liu L, Zong C, Li B, Shen D, Tang Z, Chen J, Zheng Q, Tong X, Gao C, Wang J: The interaction between beta1 integrins and ERK1/2 in osteogenic differentiation of human mesenchymal stem cells under fluid shear stress modelled by a perfusion system. J Tissue Eng Regen Med 2012 [Epub ahead of print].

55. Ward DF Jr, Williams WA, Schapiro NE, Weber GL, Christy SR, Salt M, Klees RF, Boskey A, Plopper GE: Focal adhesion kinase signaling controls cyclic tensile strain enhanced collagen I-induced osteogenic differentiation of human mesenchymal stem cells. Mol Cell Biomech 2007, 4:177-188.

56. Cukierman E, Pankov R, Stevens DR, Yamada KM: Taking cell-matrix adhesions to the third dimension. Science 2001, 294:1708-1712.

57. Leucht P, Kim JB, Currey JA, Brunski J, Helms JA: FAK-Mediated mechanotransduction in skeletal regeneration. PLoS One 2007, 2:e390.

58. Davenport JR, Yoder BK: An incredible decade for the primary cilium: a look at a once-forgotten organelle. Am J Physiol Renal Physiol 2005, 289:F1159-F1169.

59. Kottgen M, Buchholz B, Garcia-Gonzalez MA, Kotsis F, Fu X, Doerken M Boehlke C, Steffl D, Tauber R, Wegierski T, Nitschke R, Suzuki M, KramerZucker A, Germino GG, Watnick T, Prenen J, Nilius B, Kuehn EW, Walz G: TRPP2 and TRPV4 form a polymodal sensory channel complex. J Cell Biol 2008, 182:437-447.

60. Kwon RY, Temiyasathit S, Tummala P, Quah CC, Jacobs CR: Primary ciliumdependent mechanosensing is mediated by adenylyl cyclase 6 and cyclic AMP in bone cells. FASEB J 2010, 24:2859-2868.

61. Serra R: Role of intraflagellar transport and primary cilia in skeletal development. Anat Rec (Hoboken) 2008, 291:1049-1061.

62. Haycraft CJ, Zhang Q, Song B, Jackson WS, Detloff PJ, Serra R, Yoder BK: Intraflagellar transport is essential for endochondral bone formation. Development 2007, 134:307-316.

63. Malone AM, Anderson CT, Tummala P, Kwon RY, Johnston TR, Stearns T, Jacobs CR: Primary cilia mediate mechanosensing in bone cells by a calciumindependent mechanism. Proc Natl Acad Sci U S A 2007, 104:13325-13330.

64. Hoey DA, Kelly DJ, Jacobs CR: A role for the primary cilium in paracrine signaling between mechanically stimulated osteocytes and mesenchymal stem cells. Biochem Biophys Res Commun 2011, 412:182-187.

65. Hoey DA, Tormey S, Ramcharan S, O'Brien FJ, Jacobs CR: Primary cilia-mediated mechanotransduction in human mesenchymal stem cells. Stem cells 2012, 30:2561-2570.

66. Campbell JJ, Bader DL, Lee DA: Mechanical loading modulates intracellular calcium signaling in human mesenchymal stem cells. $J$ App Biomater Biomech 2008, 6:9-15.

67. Rodda SJ, McMahon AP: Distinct roles for Hedgehog and canonical Wnt signaling in specification, differentiation and maintenance of osteoblast progenitors. Development 2006, 133:3231-3244.

68. Kim JB, Leucht P, Lam K, Luppen C, Ten Berge D, Nusse R, Helms JA: Bone regeneration is regulated by wnt signaling. J Bone Miner Res 2007, 22:1913-1923. 
69. Baksh D, Tuan RS: Canonical and non-canonical Wnts differentially affect the development potential of primary isolate of human bone marrow mesenchymal stem cells. J Cell Physiol 2007, 212:817-826.

70. Arnsdorf EJ, Tummala P, Jacobs CR: Non-canonical Wnt signaling and $\mathrm{N}$-cadherin related beta-catenin signaling play a role in mechanically induced osteogenic cell fate. PLoS One 2009, 4:e5388.

71. Tschumperlin DJ, Liu F, Tager AM: Biomechanical regulation of mesenchymal cell function. Curr Opin Rheumatol 2013, 25:92-100.

72. Dupont S, Morsut L, Aragona M, Enzo E, Giulitti S, Cordenonsi M, Zanconato F, Le Digabel J, Forcato M, Bicciato S, Elvassore N, Piccolo S: Role of YAP/TAZ in mechanotransduction. Nature 2011, 474:179-183.

73. Jacobs CR, Yellowley CE, Davis BR, Zhou Z, Cimbala JM, Donahue HJ: Differential effect of steady versus oscillating flow on bone cells. J Biomech 1998, 31:969-976.

74. Ponik SM, Triplett JW, Pavalko FM: Osteoblasts and osteocytes respond differently to oscillatory and unidirectional fluid flow profiles. J Cell Biochem 2007, 100:794-807.

75. Sakai D, Kii I, Nakagawa K, Matsumoto HN, Takahashi M, Yoshida S, Hosoya T, Takakuda K, Kudo A: Remodeling of actin cytoskeleton in mouse periosteal cells under mechanical loading induces periosteal cell proliferation during bone formation. PLOS One 2011, 6:e24847.

76. Xu BY, Song GB, Ju Y, Li X, Song YH, Watanabe S: RhoA/ROCK, cytoskeletal dynamics, and focal adhesion kinase are required for mechanical stretch-induced tenogenic differentiation of human mesenchymal stem cells. J Cell Physiol 2012, 227:2722-2729.

77. Espinha LC, Jacobs CR, Hoey D: Oscillatory fluid flow influences the number of microtubules attached to the base of primary cilia [abstract]. J Biomech 2012, 45:5446.

78. Alenghat FJ, Nauli SM, Kolb R, Zhou J, Ingber DE: Global cytoskeletal control of mechanotransduction in kidney epithelial cells. Exp Cell Res 2004, 301:23-30.

79. Shi Y, Fu Y, Tong W, Geng Y, Lui PP, Tang T, Zhang X, Dai K: Uniaxial mechanical tension promoted osteogenic differentiation of rat tendonderived stem cells (rTDSCs) via the Wnt5a-RhoA pathway. I Cell Biochem 2012, 113:3133-3142.

80. Huang $Y$, Jia X, Bai $K$, Gong $X$, Fan Y: Effect of fluid shear stress on cardiomyogenic differentiation of rat bone marrow mesenchymal stem cells. Arch Med Res 2010, 41:497-505.

81. Bai $K$, Huang $Y$, Jia $X$, Fan $Y$, Wang W: Endothelium oriented differentiation of bone marrow mesenchymal stem cells under chemical and mechanical stimulations. J Biomech 2010, 43:1176-1181.

doi:10.1186/scrt318

Cite this article as: Chen and Jacobs: Mechanically induced osteogenic lineage commitment of stem cells. Stem Cell Research \& Therapy 2013 4:107. 